Japan. J. Med. Sci. Biol., 42, 149-161, 1989.

REVIEW

\title{
SEROLOGICAL DIAGNOSIS OF TRICHINOSIS BY ENZYME LINKED IMMUNOSORBENT ASSAY (ELISA)
}

Yutaka NAKAI and Keiji OGIMOTO

Department of Animal Science, Ibaraki University, Ami, Inashiki, Ibaraki 300-03 and Department of Animal Science, Tohoku University, Sendai, Miyagi 980

(Received June 13, 1989. Accepted February 7, 1990)

\section{INTRODUCTION}

Trichinosis, known as a dangerous zoonosis for more than a century, is caused by infection with Trichinella spiralis (Nematoda, Trichurata, Trichuroidea, Trichinellidae). The disease is still present worldwide, especially in America, Europe and Southeast Asia.

For the prevention of human trichinosis, European countries require, by law, inspection of each pig at the time of slaughter. In 1878, when regulatory measures began, the incidence of the swine infection in Germany was $0.05 \%$, and it declined to $0.00003 \%$ in 1966 . In the U. S., however, pigs have not been inspected for trichinosis except during 1891-1906, and in 1971 the rates of infection of grain-fed hogs and garbage-fed hogs were still $0.125 \%$ and $0.5 \%$, respectively (1).

European countries approve of two kinds of inspection methods, namely, the microscopic detection of larvae from the muscles (the trichinoscope method) and the detection of $T$. spiralis larvae after enzyme digestion of the muscles of autopsied animals (the muscle-digestion method). The disadvantages of these

中井 裕 (茨城大学農学部家畜保健学教室 茨城県稲敷郡阿見町阿見3998)

扇元敬司(東北大学農学部家畜衛生学教室＼cjkstart宮城県仙台市堤通雨宮町1-1) 
direct methods are that muscle sampling and subsequent processing are time consuming and that the infection can be detected only after a sufficient number of larvae have migrated to the muscle. Furthermore, although the direct methods can also be applied for the diagnosis of human cases after biopsy or autopsy, the methods are far from ideal because of low sensitivity and difficulty of sampling.

Many immunological diagnostic methods have been attempted to resolve the above disadvantages, namely, complement-fixation (CF) test, indirect hemagglutination (IHA) test, bentonite flocculation (BF) test, indirect immunofluorescent antibody (IIF) test, microprecipitin (larva precipitin) (MP) test, counter immuno electrophoresis (CIE) test, enzyme-linked immunosorbent assay (ELISA), etc.

Since the characteristics of these methods have been extensively discussed in previous reviews (2-5), they will be only briefly mentioned here. The CF test is one of the first methods to be used for the diagnosis of trichina infection (6). However, the CF test is unsatisfactory for diagnosis because of great differences among results obtained by laboratories on sensitivity and specificity, and of time consumption and cost (3). The IHA test is more sensitive than the CF test (7). The disadvantage of the former is that antigen-sensitized sheep red blood cells have to be prepared freshly or periodically and hence the method is not suitable for commercial use. The BF test is an improved version of the IHA test (8), relying on bentonite (a type of clay) instead of red blood cells. It is more convenient than the IHA test and more sensitive than the CF test (9) and is used by many laboratories in the U. S., including the Centers for Disease Control in Atlanta (10). After improvement of antigens and procedures, the BF test may become one of the standard diagnostic methods which is comparable with ELISA. The IIF test was used with good results in many laboratories (11-14). Although the IIF test is more sensitive than the CF or the IHA test, it has problems in specificity. In the IIF test, cross-reactions between $T$. spiralis and other parasites were reported (15). The MP test is a simple direct method. Precipitates which are formed around the oral orifices of living larvae after incubation in patient sera are observed microscopically (16-18). The test is simple to perform and has a high degree of specificity (18). The disadvantages of the test are on the preparation of live larvae, specialized laboratory techniques and heavy time consumption. The CIE test was originally developed as a screening test for the hepatitis antigen (19). The test has been applied to the diagnosis of trichinosis and is sensitive and specific enough for routine use (10,20-22). However, the test requires larger amounts of antigen and serum and is more expensive than ELISA. 
Compared with these immunological methods, ELISA is highly sensitive and specific and has many advantages, namely, low cost, possibility of standardization of methods and reagents, rapidity and amenability to automation (23). At the present time, ELISA is one of the most attractive methods for the diagnosis of both swine and human trichinosis.

\section{ELISA for diagnosis of swine trichinosis}

ELISA for serological diagnosis of swine trichinosis was attempted first by Ruitenberg's group of the Netherlands (24-26). They performed ELISA by using a crude worm extract (CWE). Supernatant $(45,000 \times \mathrm{g}, 1 \mathrm{hr})$ of sonic disintegrated muscle larvae of $T$. spiralis was used as an antigen. The antigen was coated in polystyrene test tubes and allowed to react with swine sera. The reaction was detected spectrophotometrically after adding horseradish peroxidase conjugated sheep IgG anti-porcine IgG and the substrate containing 5-amino-2hydroxybenzoic acid (5AS) and hydrogen peroxide. In sera of SPF pigs infected with $T$. spiralis larvae, specific antibodies were detected by the ELISA from 3 days after infection. Ruitenberg, van Knapen and their colleagues strove to improve the ELISA to be the reliable diagnosis by using the CWE antigen consistently. In the laboratory trials, they observed that the ELISA was more sensitive than either IHA, IIF or muscle-digestion methods in pigs infected experimentally with $T$. spiralis (27-32). To assess the reliability of the method, they planned a joint investigation with the European Economic Community (EEC) Working Group on Trichinosis. They, the personnel at the National Institute of Public Health in The Netherlands, collected samples of sera and diaphragms of swine infected with $T$. spiralis larvae and sent the samples to research institutes in EEC countries. These samples were examined by ELISA, IIF, trichinoscopy and the digestion method at each institute. Based on the number of institutes which obtained positive results by each method, ELISA and IIF were concluded to be more sensitive than trichinoscopy and the digestion method. Although ELISA and IIF were comparable each other with regard to the reliability in general, the former was more sensitive than the latter during the onset of the infection (17 days after infection) in pigs with a large number of larvae (33). Moreover, the working group extended the period of sampling of the sera and muscles of the infected pigs. This enabled them to find that positive results were detected sensitively by ELISA from an earlier stage of infection of 
swine trichinosis (2-3 weeks after infection) (34) to the later stage (40 weeks after infection) (35) than by the direct methods. The group concluded that the ELISA with CWE is one of the best methods for monitoring experimental infection and for the epidemiological survey of swine trichinosis. However, the group considered that ELISA could not be the alternative method for the "EEC approved" inspection method in abattoirs (i.e. the muscle-digestion method and trichinoscopy), because ELISA could not discriminate between past and present exposures to the parasite, in other words, between absence and presence of infective larvae in the muscle at the abattoir (33). The extensive work of the EEC Working Group clearly shows that ELISA using the crude antigen is a valuable method for monitoring experimental swine trichinosis and ELISA has possibility to be applied as a standard method in laboratories in different countries.

Many authors desired more accurate results of ELISA with high sensitivity and reliability and tried to improve the ELISA method by using purified antigens. Saunders et al. (36) purified the antigen from crude extract of muscle larvae with ultrafiltration membranes. They examined 56 farm-raised pigs from which over one cyst of larva per gram of muscle were detected by the digestion method. This density of the worm in the muscle is thought to be the minimum dose sufficient to infect humans with the parasite through pork meat. All sera of the pigs were ELISA positive. They also investigated sera of packing-house pigs which were negative by the muscle-digestion method. The ELISA using the purified antigen detected one positive pig out of 360 samples. On the other hand, ELISA using CWE antigen showed 28 positive cases out of 180 of serum samples in the same group of the packing house. The smaller number of positive pigs in the ELISA with the ultrafiltration-purified antigen is considered to have been caused by higher specificity of the method rather than lower sensitivity.

Taylor et al. (37) fractioned CWE with Sephadex G-200 and prepared a purified antigen. They performed ELISA with the purified antigen on sera of 100 slaughterhouse pigs which were negative by both the IIF and muscle-digestion methods but were positive by ELISA with CWE. The ELISA with the purified antigen showed significantly lower values than did that with CWE. Their work indicated that the Sephadex-purified antigen was useful to eliminate false positives in ELISA. The fractionation with Sephadex is one of the convenient methods for purification of the antigen.

In place of CWE, Gamble et al. (38) used filtrate of the culture medium after $72 \mathrm{hr}$ of cultivation of muscle larvae as an antigen. They called it excretorysecretory (ES) antigen. They applied the antigen to sample sera of 176 farm- 
raised pigs negative in the muscle-digestion test and 35 experimentally-infected pigs. The ELISA with ES antigen distinguished clearly two groups, while ELISA with CWE antigen did not. They tested also 37 samples from naturally-infected pigs with worm burdens ranging from 0.01 to 2.30 larvae per gram (LPG) of the diaphragm. The ELISA with ES antigen detected positive sera from the pigs with as low as 0.01 LPG. By using the same method, however, false-negative reactions were observed in serum samples of two pigs with 0.02 and 0.03 LPG. Since sera from the pigs experimentally infected with 500 muscle larvae showed positivity later in the course of infection than did the sera from the pigs receiving 10,000 larvae, they speculated that the false-negative reaction was caused by the low antibody titer of the pigs infected with a few larvae.

Murrel et al. (39) performed ELISA using the Gamble's ES antigen on sera of 305 farm-raised pigs. Of 162 muscle digestion-positive pigs, 7\% were ELISA negative and $8 \%$ of 143 muscle digestion-negative animals were ELISA positive. This may imply that the ELISA with ES antigen gave false-positive and falsenegative results. Smith and Snowdon (40) also used the Gamble's ES antigen for ELISA. They infected six pigs with a low dose of T. spiralis nativa, a subspecies of $T$. spiralis, and demonstrated that sera from five pigs of them were positive by ELISA using the ES antigen of $T$. spiralis spiralis. The result indicated that exposure to such different subspecies of $T$. spiralis undoubtedly gave rise to false positive reaction in ELISA with ES antigen. Smith (41) detected T. spiralis spiralis larvae from the muscles of five out of 1,009 swine of which the sera were negative in ELISA using the ES antigen. He also observed low ELISA titers of sera from pigs which had been infected experimentally with 3,000 larvae, a dose enough to detect the larvae in the muscle. From these results, when the ES antigen was used, there were still problems in the specificity and reliability of ELISA. To improve ELISA as a precise method, a much more specific antigen than the ES antigen should be applied.

Despommier and his colleagues (42) revealed cross-immunogenicity between the ES antigen and secretory granules of stichocytes in the stichosome. The stichosome is a characteristic organ that lies dorsal to the esophagus of all larval and adult stages of Trichuroidea. Despommier et al. (43) prepared an antigen of a large particle fraction derived from stichocytes in the supernatant of homogenated muscle larvae after centrifugation $(100,000 \times \mathrm{g}, 1 \mathrm{hr})$. The fraction was named 'S3'. They prepared hyperimmune sera from rabbits infected orally with $T$. spiralis larvae, and performed immunoaffinity chromatography by coupling the rabbit hyperimmune IgG-IgA to Sepharose-6B. The specific antigen 
in S3 was purified by affinity chromatography (44). The antigen was applied to ELISA $(45,46)$. The ELISA with the affinity-purified antigen detected experimentally infected pigs as positive earlier than did the muscle-digestion test and showed a higher sensitivity than the ELISA using the S3 antigen. Then, ELISA with either of the two antigens, the affinity-purified antigen and the S3 antigen, were applied to sera of 100 slaughter-house pigs of negative in the muscle-digestion test and to those of 75 experimentally infected pigs. In ELISA using the S3 antigen, ELISA values of the sera of 20 out of 100 muscle digestionnegative pigs were equal to or higher than those of the lowest value of sera of muscle digestion-positive pigs. On the contrary, in ELISA with the affinitypurified antigen, the values of the sera from muscle digestion-negative pigs were significantly lower than those of the sera of the positive pigs. Therefore, the sera from two groups of pigs were completely distinguished from each other in ELISA with the affinity-purified antigen. Moreover, 1,130 serum samples from pigs in Puerto Rico were tested by ELISA $(45,46)$. Puerto Rico has no endogenous trichinosis, and all 1,130 pigs were negative in the muscle-digestion test. In ELISA with the affinity-purified antigen, ELISA values from the pigs were significantly lower than those from 87 experimentally infected pigs. Their work shows that ELISA with the affinity-purified antigen distinguishes between muscle digestion-negative and positive pigs, and that the ELISA using affinitypurified antigen is more reliable than that with either crude or partially purified antigen.

Rapic et al. (47) used the affinity-purified antigen of Despommier's group (44) for ELISA on serum samples of 1,000 pigs from rural farms in trichinosis endemic areas. Muscle larvae were detected from 16 pigs and serum samples from these pigs were all positive by ELISA using each of the purified antigen, the ES antigen and the crude worm-extract antigen. However, the ELISA with the ES antigen and the crude antigen also showed the positivity in the sera of five and seven pigs other than the above 16 pigs, respectively. From these five and seven pigs, no larvae were detected. Their work suggests that the results of ELISA with affinity-purified antigen correlate more closely with those of the muscle-digestion method than those of ELISA with ES or crude antigen.

Despommier's group (48) obtained monoclonal antibodies against the affinity-purified antigen. Hybridomas were prepared by fusing mouse myeloma cell line with the spleen cells from the mice infected orally with the larvae. Three hybridoma cell lines were obtained after testing supernatant fluids for positivity by ELISA using the purified antigen. They coupled the monoclonal antibodies to 
immunoaffinity columns, and isolated $50 / 55 \mathrm{kDa}, 48 \mathrm{kDa}$ and $37 \mathrm{kDa}$ polypeptides. The $50 / 55 \mathrm{kDa}$ and $48 \mathrm{kDa}$ were located in the $\alpha$ and $\beta$-stichocytes, respectively, both of which were secretory protein of the muscle larvae. In particular, the $48 \mathrm{kDa}$ protein possessed immunogenicity to prevent the infection of mice with the larvae (48). These antigens are very attractive in application for vaccination and also for ELISA.

On the other hand, Gamble's group also obtained monoclonal antibodies against the ES antigen (49). Their monoclonal antibodies were reactive specifically with the ES antigen. The immunoaffinity columns coupled with the monoclonal antibodies isolated $53 \mathrm{kDa}$ and $49 \mathrm{kDa}$ proteins of the stichocytes. Although sera of 11 out of 35 slaughterhouse pigs which were negative for the muscle-digestion test showed false positive reaction in the ELISA using the crude antigen, all sera from the pigs were negative in the ELISA using the antigen purified with the monoclonal antibody column (49). With the monoclonal purified antigen, the ordinary ELISA method was compared with a competitive ELISA method which is based on competitive binding between the swine serum antibody and the monoclonal antibody (50). In both ELISA methods, 30 serum samples from naturally infected cases which were positive in the muscle-digestion test showed significantly higher values than did 10 samples from farm-raised pigs which were negative in the digestion test.

Reliability and sensitivity of the ELISA using the antigen purified with the monoclonal antibody column should be established by applying it for expanded screening on farms and in slaughter houses. If satisfactory results are obtained by the ELISA, it will be a standard method for detecting the natural and experimental infection.

\section{ELISA for diagnosis of human trichinosis}

Ruitenberg's group applied ELISA to serodiagnosis of human trichinosis $(24,51)$. The reactions between patient sera and supernatant $(25,000 \times \mathrm{g}, 15 \mathrm{~min})$ of homogenated muscle larvae were tested with alkaline phosphatase-labeled anti-human Ig rabbit Ig and p-nitrophenyl phosphate in diethanolamine buffer. They compared OD values of the ELISA and titers of IHA and IIF of the serum samples from the patients at various intervals after onset of clinical sign. All three methods detected significant amounts of antibodies in the sera from the patients even in 2 years after infection. Nevertheless, the sensitivity of ELISA 
was comparable to that of IHA and higher than that of IIF. The group's work first showed that the specific antibody in the sera of patients of trichinosis could be detected by ELISA. Apart from the T. spiralis antibody, they tested also the reactivity of the sera from five persons infected with some species of filariae by the ELISA. Positive ELISA values were shown with the sera from four of the five persons. These results suggest a cross reactivity between the $T$. spiralis antigen and those of some species of filariae. As the group discussed, purification of the antigen is needed to remove the cross reactivity in the sera from individuals infected with other nematodes.

Van Knapen et al. (52) pursued specific serum, IgG, IgM, IgA and IgE of 58 patients of trichinosis by ELISA using CWE antigen. These patients had eaten under-cooked wild boar meat containing $T$. spiralis larvae in the Netherlands, and became ill with all the symptoms of acute trichinosis. At the outbreak, IgG and IgM specific for the CWE antigen were detected from $100 \%$ and $86 \%$ of patients, respectively. These specific IgG and IgM were still detected from $52 \%$ and $47 \%$ of patients after 11 months, respectively. However, specific IgA was detected from $62 \%$ of patients after 2 months of the infection, $16 \%$ after 3 months, and only $2 \%$ after 5 months. Specific IgE was detected from only $7 \%$ of patients even at the outbreak. By IIF, 95\% of patients were positive at the outbreak and so were $32 \%$ after 11 months. Their work suggests that ELISA detecting specific IgG is more sensitive than IIF or ELISA detecting other Igs.

In Asia, Au et al. (53) investigated serum samples of 18 patients of acute trichinosis by radioimmunoassay (RIA), IHA, ELISA using CWE antigen after one week of the infection. The epidemic of trichinosis occurred in Hong Kong among the Gurkha soldiers stationed locally. Their eating of under-cooked pork in barbecues was suspected of being the cause of the outbreak. By ELISA, specific IgE and IgM were detected in $100 \%$ of patients, and specific IgG in $94 \%$. By RIA or IHA, specific IgG was shown in $94 \%$ of patients. Although no specific IgM nor IgE by ELISA nor IgG by IHA was detected in the samples of 187 healthy Gurkha soldiers, specific IgG was detected by RIA and ELISA from $0.5 \%$ and $5 \%$ of them, respectively. On the other hand, serum samples of 100 healthy Chinese were negative in RIA for IgG and ELISA for IgM, IgG and IgE. Therefore, there might have been some Gurkha soldiers who had acquired trichinosis already. In the same article (53), Au et al. stated also that only specific IgG was detected by ELISA in $9 \%$ of 35 Chinese who had records of other parasitic infections, e.g. clonorchiasis, ascariasis, trichomoniasis, etc. They reached the conclusion that detecting IgE or IgM specific for trichinosis by ELISA is the most suitable for 
diagnosis. Their results were obtained in patients during the first week, which did not coincide with those of van Knapen who showed that ELISA detecting specific IgG was the most sensitive in case of sera of patients in the first month of illness. It seems possible that the classes of Ig specific for trichinosis change in the first month of illness. In order to reveal which class of Ig is predominant in the infection or which class of Ig is suitable for being detected by ELISA, more detailed investigation should be performed by monitoring specific Igs for a long period of the disease from just after the outbreak of the infection.

In Japan, Kobayashi et al. (54) investigated the sera of 474 cases of suspected human trichinosis by the double diffusion test in agar (DD), CF and ELISA using CWE. These suspected individuals had eaten uncooked imported bear meat in Mie Prefecture. In ELISA, 74 sera were positive, and in CF and DD, 35 and 19 sera were positive, respectively. In all, the sera from 77 persons showed positivity in these methods. These results suggest that ELISA is the most sensitive among the three methods. They also prepared a partially purified antigen (S3) derived from stichocytes by the method of Despommier and Lacetti (43) and compared the reactivity of S3 with that of CWE against the sera from the positive cases. Of 50 cases positive in ELISA with CWE antigen after 33-52 days of the infection, ELISA with S3 detected 35 positive cases. However, the titers in ELISA with S3 were higher than those in ELISA with CWE after 98-188 days of the infection. These results may indicate a higher specificity of the ELISA using S3. Furthermore, when S3 antigen was used, no positive reaction was observed in 20 serum samples from other helminthiasis patients, while eight of the 20 sera were positive with CWE antigen. Their work indicates that the use of purified antigen improves the specificity of ELISA in human cases as was shown in swine cases.

Nakai et al. (55) prepared crude antigens extracted from larvae of three strains of T. spiralis isolated in Japan, Thailand and Poland, and ELISA using these antigens was applied to serum samples taken from a patient of trichinosis in Thailand. At the onset of the infection, the highest ELISA value was observed when the Thailand strain antigen was used, and the second highest value was found with the Japanese one. After 265 days of the infection, the titer of the serum decreased against the Thailand strain antigen,but not against the Japanese one. From their results, it is speculated that there exist at least two types of antibodies in T. spiralis infection, i.e. strain-specific and species-specific antibodies. The strain-specific antibodies might disappear earlier than speciesspecific antibodies in the course of recovery of the disease. Strain-specific 
antigens of $T$. spiralis must be present. The antigenic differences among strains of $T$. spiralis should be noticed when the serodiagnosis for trichinosis is carried out.

\section{ELISA for diagnosis of trichinosis in the future}

In many countries, pigs are examined for the existence of pathogens when they are slaughtered. Though such direct diagnostic methods as the trichinoscopic method or the muscle-digestion method are effective in reducing the incidence of human trichinosis, they unfortunately can detect trichina larvae only in meats with a high burden of worms. The most desirable method for diagnosis of trichinosis is such that can detect any trichina infection even if it is a light one. Moreover, rapid and convenient methods are required at slaughterhouses. Therefore, high sensitivity, high amenability to automation and low cost are all essential conditions for inspection for swine trichinosis. From this point of view, ELISA has many advantages in that it is rapid and sensitive, can be automatized, is sufficient even with a small amount of sera and reagents, and is economical. ELISA has a high specificity, the degree of which depends upon the purity of the antigen and the Ig class detected. Accordingly, ELISA will be appropriate for screening of trichinosis among farm and wild animals.

The ELISA using crude antigens has been reported to have low reliability and low reproducibility in diagnosis of swine trichinosis at the time of slaughter (36-38); however, these problems can be overcome by ELISA using highly specific antigens purified by immunoaffinity chromatography as shown in two reports $(48,50)$. Minimal cross reactivity with other parasitic diseases and higher reliability may be obtained by the ELISA with affinity-purified antigens. When the antigen purified by immunoaffinity chromatography with polyclonal or monoclonal antibodies is standardized, reliable and reproducible results can be obtained in any laboratory in the world. This will be understood more clearly after further improvement and application of the ELISA.

In human trichinosis, false positivity due to cross reaction with other parasitic infection interferes in the results of ELISA $(51,53,54)$. The problem will be solved by a combination of clinical diagnosis and serodiagnosis by ELISA using a purified antigen with high specificity. On the other hand, since antigenic differences of trichina strains were suggested (55-60), ELISA using a highly purified antigen with a single or a few epitopes would not detect patients infected 
with some of $T$. spiralis strains with the antigenicity different from the above ELISA antigen. Therefore, if ELISA is applied to the diagnosis, such antigens that are reactive with a wide range of stains of $T$. spiralis should be used.

Problems relating to preparation and purification of antigens and to antigenic differences of strains of $T$. spiralis would be solved in the near future. ELISA should be a powerful standard method in diagnosis and screening of trichinosis of swine, wild animals and human.

\section{ACKNOWLEDGEMEN'TS}

We thank Ms. Patricia Walters for reviewing the manuscript.

\section{REFERENCES}

1. Leighty, J. C. (1983): Food Technol., 95-97.

2. Despommier, D. D. (1986): In K. W. Walls and F. M. Schauty (eds.), Immunodiagnosis of parasitic diseases, Vol. 1, p.163-181. Academic Press, London and New York.

3. Kagan, I. G. and Norman, L. G. (1970): In S. E. Gould (ed.), Trichinosis in man and animals. 1st ed., p.222-268. Charles C. Thomas Publisher, Springfield.

4. Kazura, J. W. (1982): In S. Cohen and K. S. Warren (eds.), Immunology of parasitic infections. 2nd ed., p.654-675, Blackwell Science Publisher, Oxford.

5. Steele, J. H. (1981): In J. H. Steele (ed.), CRC handbook series in zoonoses. Section C: Parasitic zoonoses. Vol. II, p.293-324. CRC Press, Boca Raton, Florida.

6. Witebsky, E., Wels, P. and Heide, A. (1942): New York J. Med., 42, 431-435.

7. Prince, S. G. and Weiner, L. M. (1956): Amer. J. Clin. Pathol., 26, 12611269.

8. Bozicevitch, J., Tobie, J. E, Thomas, E. H., Hoyem, M. H. and Ward, S. B. (1951): Publ. Hlth Rep., 66, 806-814.

9. Kozar, M., Koar, Z. and Karmanska, K. (1964): Wiad. Parazyt., 10, 717-737.

10. Norman, L. and Kagan, I. (1975): Bol. Chil. Parasitol., 30, 58-64.

11. Brzosko, W., Gancarz, Z. and Nowoslawski, A. (1965): Exptl. Med. Microbiol., 17, 355-365. 
12. Labzoffsky, N. A., Baratawidjaja, R. K., Kurtunen, E., Lewis, F. N., Kavelman, D. A. and Morrissey, L. P. (1964): Can. Med. Assoc. J., 90, 920921.

13. Ljungstrom, I. (1983): In W. C. Campbell (ed.), Trichinella and trichinosis. p. 403-424. Plenum, New York.

14. Sulzer, A. J. (1965): J. Parasitol., 51, 717-721.

15. Saathoff, M., Kasper, M. and Demmer, H. (1978): Dtsch. Med. Wochenschr., 103, 1606-1611.

16. Jackson, G. J. (1959): J. Infect. Dis., 105, 97-117.

17. Oliver-Gonzalez, J. (1941): J. Infect. Dis., 69, 254-270.

18. Roth, H. (1941): Acta Pathol. Microbiol. Scand., 18, 160-167.

19. Gocke, D. J. and Howe, C. (1970): J. Immunol., 104, 1031-1032.

20. Barret-Connor, E., Davis, C. F., Hamburger, R. N. and Kagan, I. G. (1976): J. Infect. Dis., 133, 473-477.

21. Despommier, D. D., Muller, M., Jenks, B. and Fruitstone, M. (1974): Amer. J. Trop. Med. Hyg., 23, 41-44.

22. Tsotsos, A. S. and Corbitt, G. (1973): J. Immunol. Methods, 3, 53-62.

23. Ruitenberg, E. J., van Amstel, J. A., Brosi, B. J. M. and Steerenberg, P. A. (1977): J. Immunol. Methods, 16, 351-359.

24. Ljungstrom, I., Engvall, E. and Ruitenberg, E. J. (1974): Parasitol. BSP Proc. 69, XXIV.

25. Ruitenberg, E. J., Steerenberg, P. A., Brosi, B. J. M. and Buys, J. (1974): Bull. Wld. Hlth. Org., 51, 108-109.

26. Ruitenberg, E. J., Steerenberg, P. A., Brosi, B. J. M. and Buys, J. (1975): Wiad. Parazytol., 21, 747-751.

27. Ruitenberg, E. J., Ljungstrom, I., Steerenberg, P. A. and Buys, J. (1975): Ann. New York Acad. Sci., 254, 296-303.

28. Ruitenberg, E. J., Steerenberg, P. A., Brosi, B. J. M. and Buys, J. (1976): J. Immunol. Methods, 10, 67-83.

29. van Knapen, F., Framstad, K. and Ruitenberg, E. J. (1976): J. Parasitol., 62, 332-333.

30. Ruitenberg, E. J. and van Knapen, F. (1977): Vet. Parasitol., 3, 317-326.

31. Ruitenberg, E. J. and van Knapen, F. (1977): J. Infect. Dis., 136, S267-273.

32. Ruitenberg, E. J. and Buys, J. (1979): Vet. Parasitol., 5,73-78.

33. van Knapen, F., Franchimont, J. H., Ruitenberg, E. J., Baldelli, B., Bradley, J., Gibson, T. E., Gottal, C., Henriksen, S. A., Kohler, G., Skovgaard, N., Soule, C. and Taylor, S. M. (1980): Vet. Parasitol., 7, 109-121.

34. van Knapen, F., Franchimont, J. H., Ruitenberg, E. J., Andre, P., Baldelli, B., Gibson, T. E., Gottal, C., Henriksen, S. A., Kohler, G., Roneus, O., Skovgaard, N., Soule, C., Strickland, K. L. and Taylor, S. M. (1981): Vet. Parasitol., 9, 117-123.

35. van Knapen, F., Franchimont, J. H., Ruitenberg, E. J., Andre, P., Baldelli, B., Gibson, T. E., Henriksen, S. A., Kohler, G., Roneus, O., Skovgaard, N., 
Soule, C., Strickland, K. L., Taylor, S. M., Thomsen, D.U. and Wolff, F. (1984): Vet. Parasitol., 16, 167-171.

36. Saunders, G. C., Clinard, E. H., Bartlett, M. L. and Sanders, W. M. (1977): J. Infect. Dis., 136, S258-266.

37. Taylor, S. M., Kenny, J., Mallon, T. and Davidson, W. B. (1980): Zbl. Vet. Med., B27, 764-772.

38. Gamble, H. R., Anderson, W. R., Graham, C. E. and Murrell, K. D. (1983): Vet. Parasitol., 13, 349-361.

39. Murrell, K. D., Anderson, W. R., Schad, G. A., Hanbury, R. D., Kazacos, K. R., Gamble, H. R. and Brown, J. (1986): Amer. J. Vet. Res., 5, 1046-1049.

40. Smith, H. J. and Snowdon, K. E. (1987): Can. J. Vet. Res., 51, 413-414.

41. Smith, H. J. (1987): Can. J. Vet. Res., 51, 194-197.

42. Despommier, D. D. and Muller, M. (1976): J. Parasitol., 62, 775-785.

43. Despommier, D. D. and Lacetti, A. (1981): Exptl. Parasitol., 51, 279-295.

44. Despommier, D. D. and Lacetti, A. (1981): J. Parasitol., 67, 332-339.

45. Seawright, G. L., Sanders, W. M. and Bryson, M. (1981): Adv. Exptl. Med. Biol., 137, 145-168.

46. Seawright, G. L., Despommier, D., Zimmermann, W. and Isenstein, R. S. (1983): Amer. J. Trop. Med. Hyg., 32, 1275-1284.

47. Rapic, D., Dzakula, N. and Matic-Piantanida, D. (1986): Vet. Parasitol., 21, 285-289.

48. Silberstein, D. S. and Despommier, D. D. (1984): J. Immunol., 132, 898-904.

49. Gamble, H. R. and Graham, C. E. (1984): Vet. Immunol. Immunopathol., 6, 379-389.

50. Gamble, H. R. and Graham, C. E. (1984): Amer. J. Vet. Res., 45, 67-74.

51. Engvall, E. and Ljungstrom, I. (1975): Acta Pathol. Microbiol. Scand., 83, 231-237.

52. van Knapen, F., Franchimont, J. H., Verdonk, A. R., Stumpf, J. and Undeutsch, K. (1982): Amer. J. Trop. Hyg., 31, 973-976.

53. Au, A. C. S., Ko, R. C., Simon, J. W., Ridell, N. J., Wong, F. W. T. and Templer, M. J. (1983): Trans. Roy. Soc. Trop. Med. Hyg., 77, 412-415.

54. Kobayashi, M., Niimura, M., Yokogawa, M. and Yamaguchi, T. (1987): Jpn. J. Parasitol., 36, 248-253.

55. Nakai, Y., Ogimoto, K. and Yamaguchi, 'T. (1987): Jpn. J. Parasitol., 36, 328-333.

56. Ogimoto, K. (1984): Hirosaki Med. J., 36, 233-246.

57. Ogimoto, K. (1984): Hirosaki Med. J., 36, 247-255.

58. Ogimoto, K. (1984): Hirosaki Med. J., 36, 256-273.

59. Ogimoto, K. (1984): Hirosaki Med. J., 36, 274-282.

60. Ogimoto, K. (1984): Hirosaki Med. J., 36, 283-296. 